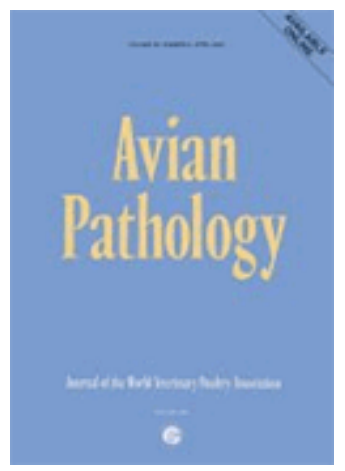

\title{
Patsturella multocida in scavenging family chickens and ducks: carrier status, age susceptibility and transmission between species
}

\begin{tabular}{|r|l|}
\hline Journal: & Avian Pathology \\
\hline Manuscript ID: & CAVP-2007-0102.R2 \\
\hline Manuscript Type: & Original Research Paper \\
\hline Duthor: & O2-Oct-2007 \\
\hline Complete List of Authors: & $\begin{array}{l}\text { Mbuthia, Paul; Faculty of Veterinary Medicine, Universithy of } \\
\text { Nairobi, Department of veterinary pathology, microbiology and } \\
\text { parasitology } \\
\text { Njagi, Lucy; University of Nairobi, Department of veterinary } \\
\text { pathology, microbiology and parasitology } \\
\text { Nyaga, Philip; University of Nairobi, Department of Veterinary } \\
\text { pathology, microbiology and parasitology } \\
\text { Minga, Uswege; Open University of Tanzania, Registrar's } \\
\text { Department } \\
\text { Kamundia, John; Kenya Agricultural Research Institute, Plant } \\
\text { Breeding Station } \\
\text { Bebora, Lily; University of Nairobi, Department of Veterinary } \\
\text { Pathology, Microbiolgy and Parasitology } \\
\text { Olsen, John; University of Copenhagen, Faculty of Life Sciences, } \\
\text { Department of Veterinary Pathology }\end{array}$ \\
\hline Keywords: & \begin{tabular}{l} 
fowl cholera, carrier, age susceptibility, transmission \\
\hline
\end{tabular} \\
\hline
\end{tabular}

\section{SCHOLARONE" Manuscripts}




\section{Cavp-2007-0102.R2 \\ Pasteurella multocida in scavenging family chickens and ducks: carrier status, age susceptibility and transmission between species}

\author{
Mbuthia, P.G. ${ }^{1}$, L.W. Njagi ${ }^{1}$ P.N. Nyaga ${ }^{1}$, L.C. Bebora ${ }^{1}$, U.Minga ${ }^{2}$ J. Kamundia ${ }^{3}$ \\ and J.E.Olsen ${ }^{4 *}$
}

$\mid \begin{aligned} & { }^{1} \text { Department of Veterinary Pathology, Microbiology } \\ & \text { Veterinary Medicine, University of Nairobi,P.O. Bo } \\ & { }^{2} \text { Administrations, The Open University of Tanzania } \\ & \text { Tanzania. }{ }^{3} \text { Plant Breeding Station, Kenya Agricultu } \\ & \text { P.O. Njoro, Kenya }{ }_{-4}^{4} \text { Department of Veterinary Path } \\ & \text { University of Copenhagen, DK- } 1870 \text { Frederiksber }\end{aligned}$

*Corresponding author

I

Department of Veterinary Pathobiology

Faculty of Life Sciences, University of Copenhagen

I Stigbøjlen 4, DK-1870 Frederiksberg C

Denmark

| Telephone +45 35332756, e-mail: jeo@ life.ku.dk

Short Title: P. multocida in scavenging chicken and ducks

| Received: 28 June 2007
Formatted: Right

Formatted: Font: Not Bold, Not

Italic

Formatted: Font: $14 \mathrm{pt}$, Complex

Script Font: $14 \mathrm{pt}$

Formatted: Left

Formatted: Font: Bold

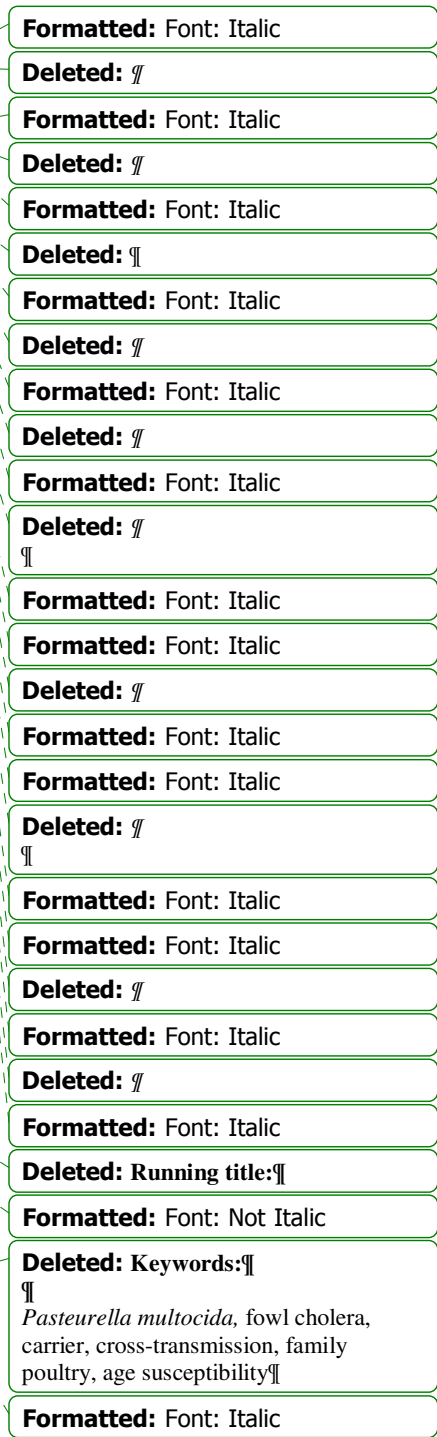


Pasteurella multocida causes fowl cholera, a highly contagious and severe disease in chicken and water fowls. The disease is not well described in less intensive production systems, including scavenging family poultry production in developing countries. $P$. multocida was isolated from $25.9 \%$ of healthy looking ducks and $6.2 \%$ of chickens from free range family poultry farms and at slaughter slabs at marked. On experimental infection with $1.2-2.0 \times 10^{8}$ organisms of the P. multocida type strain (NCTC $10322^{\mathrm{T}}$ ), 12 week-old chickens expressed fowl cholera clinical signs significantly more times (372) than those of 4,8 and 16 week chicken $(173,272,187)$ and more signs were severe. In family ducks the 8 week-old birds expressed clinical signs significantly more times (188) than those of other age groups $(117,80$, and 83$)$ and severe signs were more frequent. $P$. multocida transmitted from seeder birds $(\mathrm{n}=12)$ to sentinel birds $(\mathrm{n}=30)$, which developed clinical signs, and in some cases lesions of fowl cholera and allowed bacterial re-isolation, whether infected ducks served as seeder for chicken or chicken served as seeder for ducks. This study has documented the occurrence of P. multocida among healthy-appearing family poultry in tropical setting, and demonstrated that age 
susceptibility is highest in 12 week old family chicken and 8 week old family ducks when challenged with a low virulent strain of $P$. multocida. It has further demonstrated that cross transmission of fowl cholera may happen between family ducks and chickens and vice versa. 
Introduction

The Gram negative rod, Pasteurella multocida causes fowl cholera, which is a severe disease of poultry. It is seen either as acute or chronic forms, and the clinical signs vary depending on the form of the disease. Symptoms include depression, ruffled feathers, fever, anorexia, mucous discharge from the mouth, diarrhoea and an increased respiratory rate (Rhoades \& Rimler, 1989). Carrier birds play a major role in the transmission of fowl cholera (Christensen and Bisgaard, 2000). Deleted: $\mathbb{I}$

Free-range chicken are important livestock species for many rural families

worldwide. They are traditionally raised in a low input low output production system, where birds obtain feed by scavenging and only rarely are offered supplementary feed. Birds used in this type of production are of low genetic potential as they are often of undefined indigenous breeds that have not been subjected to a clear selection strategy. In the current publication, the term family chickens/ducks/poultry will be used for such birds. While the disease is well described from intensive production systems (see Christensen and Bisgaard, 2000 for a review), little information is available as to the presence of this disease among family chickens. Recent studies have shown family poultry may be carriers of $P$. multocida (Muhairwa et al., 2001), however, clinical cases are not frequently described, and chronic manifestations seem to be the normal clinical picture in this production system.

Family poultry are kept in a production system where different age groups mix freely during scavenging. Knowledge of the most susceptible age group is important, since disease control measures and development of health programs depend on such 
information. In chicken raised under industrial conditions, fowl cholera is mainly

diagnosed in mature chickens (Salami et al., 1989), and based on challenge of 16 and 45

week-old New Hampshire chickens with two different $P$. multocida serotypes

(Heddleston,1962) it has been generally accepted that mature birds are more susceptible than the young chickens. In support of this, Heddleston and Watko (1965), showed that 9-16 week-old New Hampshire chickens were less susceptible than 52 week old birds. In such studies, the attack criterion has been mortality, since the $P$. multocida strains used were lethal to the chickens. However, no studies on age susceptibility have been done in poultry using a less virulent strain causing clinical signs but no mortality, as seems to be the normal form of fowl cholera in family poultry.

Fowl cholera is also a recognized cause of duck mortality (Hunter and Wobeser, 1980; Pehlivanoglu et al., 1999; Amonsin et al., 2002). Fewer studies have been performed with this species. However, by use of 5, 11, 16 and 18 week-old Mallard ducks, it has been demonstrated that birds older than 11 weeks are less vulnerable to $P$. multocida infection than young ones (Hunter and Wobeser, 1980). In many developing countries, chickens and ducks are kept under scavenging conditions along side each other, and the close proximity may sustain infection if transmission can take place between species. Studies of transmission between chicken and ducks (and vice versa) have, however, not been undertaken.

The present study aimed to estimate the prevalence of carriers of $P$. multocida among healthy looking family chickens and ducks and to determine the most susceptible age group among the family chickens and ducks when challenged with a low virulent strain causing signs of chronic fowl cholera. Further it aimed to evaluate if transmission 
of $P$. multocida occurs from infected ducks to non-infected chickens and vice versa through contact. Challenge was done using the type strain of P. multocida $\left(\mathrm{NCTC} 10322^{\mathrm{T}}\right.$ - Carter serogroup A). While originally isolated from pigs, this strain causes clinical and pathological signs of chronic fowl cholera.

Materials and Methods

Samples of birds from farms and markets to determine carrier status. Eighty eight family chickens were sampled from 8 farms in three districts of Kenya. The flock sizes
Deleted: MATERIALS AND

METHODS

Formatted: Normal, Line spacing: single

Deleted: II

Samples of birds from farms and markets to determine carrier status $\mathbb{\|}$

Deleted: II

Formatted: Font: Bold ranged from 20 to 74 birds per farm. Forty seven family ducks were sampled from six farms from two districts in Kenya with a population that ranged from 18 to 85 birds per farm. Similarly, a total of 74 chickens and seven ducks were sampled from slaughterhouses and open air markets in Nairobi. The markets only receive birds from non-industrial production and upon sampling birds were ensured to originate from scavenging conditions. The birds originated from seven districts. Pre-wetted oropharyngeal and cloacal swabs were taken and transported directly to the laboratory in transport containers kept at $4^{\circ} \mathrm{C}$.

\section{Bacterial strain for experimental infections, preparation of inoculums and}

challenge $e_{\text {it }}$ The type strain of $P$. multocida (NCTC $10322^{\mathrm{T}}$ - Carter serogroup A, (BA, Oxoid Ltd., CM55, Basingstoke, Hampshire, England) with $5 \%$ citrated calf blood, incubated aerobically at $37^{\circ} \mathrm{C}$, for 24 hours to check for purity prior to preparation of the 
inoculums. The inoculums were prepared as described by Petersen et al. (2001) and each bird was inoculated intra-tracheal with $0.5 \mathrm{ml}$ culture containing $P$. multocida in brain heart infusion (BHI) broth. The size of the inoculums was determined by plate spread method and contained $1.2-2.0 \times 10^{8} \mathrm{CFU}$ per bird. Control birds were inoculated with $0.5 \mathrm{ml}$ of BHI broth and housed in different houses than the challenged birds.

Animal experimentation. Birds and general management conditions. Family chicks and Deleted: 9 ducklings, hatched from incubated eggs or bought as one day old birds from farms with indigenous flocks, were brooded and reared in an isolation house, away from other birds up to the required experimental age. They were a mixture of eco-types used for local production and could not be included with any described commercial breed. They were screened for P. multocida before used in experiments, as described under bacteriological examination below. When used for experimentation, groups were separated in different rooms located in different houses and left to acclimatize for $48 \mathrm{hr}$ before challenge. They were kept at ambient temperature on the floor, which allowed all birds to mix freely. Each room had own utensils and did not communicate with other rooms through ventilation. Birds were fed on commercial chick and grower's feed (Unga Limited, Kenya) and given water ad libitum. Experimental infections were performed with permission according to Kenyan law.

Age susceptibility studies A total of one hundred and twenty family chickens and 120 family ducks were divided into age groups $(4,8,12$, and 16 weeks) with 30 birds in each group. For each age susceptibility group, the birds were split into two groups of 15 wing 
tagged birds in each, allowing the experiment to be replicated. No statistical difference was observed between repeats, and the result section presents pooled results from both experiments. Of the 15 birds in each experimental round, 10 were infected with $P$. multocida while 5 were used as controls. They were challenged with P. multocida strain NCTC $10322^{\mathrm{T}}$ as described above and 24 hours post challenge, the birds were swabbed on oro-pharynx and cloaca to confirm the establishment or absence of $P$. multocida. On day 14 post-challenge, all birds (challenged and control) were sacrificed and examined for gross lesions and swabs were taken for bacterial isolation.

$$
\mid
$$

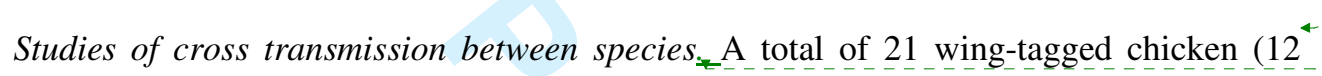
week old) and 21 wing-tagged ducks (6 week old) were used for study of cross transmission between species. In experiment number one, 6 ducks were infected intratracheal with $P$. multocida NCTC $10322^{\mathrm{T}}$ as described above (seeder birds), while 15 chickens (sentinel birds) were mixed with them under conditions that allow free mixing six hours post inoculation for contact cross infection. In the second experiment, 6 chickens were infected intra-tracheal (seeder birds), while 15 ducks (sentinel birds) were mixed with them under conditions that allowed free mixing six hours post inoculation. Five sentinel birds in each experiment were individually swabbed on the Deleted: $\mathbb{\pi}$ oropharynx and cloaca daily. The remaining 10 sentinel birds were randomly paired and sacrificed on days $1,3,5,7$, and 10 after mixing for post mortem examination. On day 14 post-infection the sentinel birds that were regularly swabbed and the seeder birds were sacrificed and examined for gross lesions and swabs taken for bacterial isolation. 
Clinical observations ${ }_{2}$ Daily observations started with birds in the control house before

proceeding to the infected house. Clinical sign observations (See Table 1 for a list of signs noted), temperature and weight measurements were done once daily, by the same person throughout the study period. The observer took 30 minutes per day per group at the same time each day for the measurements. Each day, observations were first made without disturbing the birds and later a close examination and cloacal temperature measurement were carried out while holding each bird; and its weighing was done and recorded. Observed signs were graded as severe, moderate, or mild. A sign was mild if it was observed 1-3 times during the 30 min observation period, moderate if it was observed 4-6 times and severe if it was observed 7 or more times on an individual bird during the 30 minutes of observations. Depression was mild if there was only dullness; moderate if accompanied by wing drooping; and severe when there is addition sign of dosing and/or tucked head under the wings. Ruffled feathers around the head and neck was taken as mild; when general body was affected it was taken as moderate; while with drooping wings it was regarded as severe. Cloacal temperature below $41.5^{\circ} \mathrm{C}$ was taken as normal, $41.6{ }^{\circ} \mathrm{C}$ as mild, around $42.0^{\circ} \mathrm{C}$ as moderate, and that of over $42.0^{\circ} \mathrm{C}$ was taken as severe fever.

Bacterial detection and identification ${ }_{*}$ Swabs were individually placed in two ml of sterile Phosphate Buffered Saline (PBS) and transported in a cool box to the laboratory, where it was thoroughly vortexed and $0.1 \mathrm{ml}$ was streaked on blood agar (BA) (Oxoid Ltd., CM55, Basingstoke, Hampshire, England) and incubated aerobically at $37^{\circ} \mathrm{C}$ for 24 hours for initial culture. Point-one ml of PBS was further inoculated into Pasteurella-free 
21 days old Balb/C mice by intra-peritoneal route, as described by Muhairwa et al.

(2001), to improve the bacterial recovery rate. Inoculated mice were sacrificed after 48

hours, however, severely affected mice were sacrificed on a running basis. Culture was

performed from aseptically removed liver and spleen.

Deleted: $\mathbb{I}$

Bacterial colonies from initial swabs and mice organs morphologically

Formatted: Indent: First line: 36 pt

resembling those of $P$. multocida were sub-cultured on BA and differentiated following

the procedures described elsewhere (Bisgaard and Mutters, 1986). The biochemical

reactions of the isolates were compared with those of P. multocida strain NCTC $10322^{\mathrm{T}}$

and strains were assigned to sub-species according to Mutters et al. (1985).

Post mortem procedure Post mortem examination was done as described by Bermudez

Deleted: II

II

Deleted:

Formatted: Font: Not Bold

Deleted: II

Deleted: II

proportion of infected birds at farm and at marked were compared by the chi-square

method. A p-value of 0.05 was taken as significant.

Deleted: RESULTS

Results 


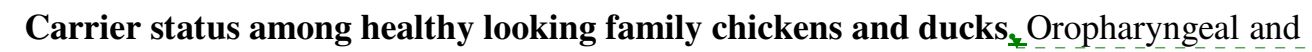
Deleted: II cloacal swab samples from 162 family chickens and 54 ducks were screened for $P$. multocida on both culture and mouse passage. They yielded 24 positive isolations. Seventeen isolates were obtained from swabbing of oropharynx and seven from cloacae. Four isolations were made by mouse passage only, while in the remaining 20 cases, isolation was done both from swabs and mice. Seven isolates were characterized as $P$. multocida subsp. multocida, 12 isolates as $P$. multocida subsp. gallicida while 5 isolates were characterized as P. multocida subsp. septica. Of the 162 chickens sampled, 10 (6.2 $\%$ ) yielded P. multocida. The proportion of infected birds at market was 9/74 (12.1\%) was significantly higher than that of birds sampled on farm $(1 / 88(1.1 \% ; \mathrm{p}<0.05))$. Of the 54 family ducks screened, 14 (25.9\%) yielded P. multocida. The proportion of infected market birds, 4/7 (57.1\%) was significantly higher than that of birds sampled on farm $(10 / 47(21.3 \% ; \mathrm{p}<0.05))$.

Age susceptibility in family chickens ${ }_{+}$Clinical signs in chicken in relation to age group. Deleted: II No birds died during the experiments although all chickens but two (16 week old) expressed clinical signs of fowl cholera at some point during the 14 days observation. The frequency of the individual clinical signs was significantly different between groups $(\mathrm{p}<0.05)$ except for diarrhea, mouth discharges and cyanosis (Table 1).

Deleted: $\mathbb{I}$ The number of clinical signs observed in each age group is listed in Table 2. The 12 week old age group expressed significantly more clinical signs (372) than the other age groups. The age susceptibility of the other age groups declined in the order 8 weeks (272 signs; $p<0.05$ compared to 4 and 16 weeks) compared to the 4 and 16 week old 
chickens that expressed almost similar number of clinical signs (173 and 187). Moreover, severe clinical signs were more often observed in the 12 week-old birds (Table 2). Control birds in all groups did not show any clinical sign throughout the observation period.

Gross lesions and P. multocida re-isolation from chickens, On post mortem the 4 weekold chickens had no visible gross lesions, while three birds in the 8 week-old group had fibrin remnants on the airsacs and thickened airsacs, one bird had fibrotic lung and another had splenomegally. Five from the 12 week-old ones had remnants of fibrin and fibrosis of the lungs and airsacs, one had necrotic liver lesions, and another one had splenomegally, while three of the 16 week-old ones had fibrosis on the lungs and airsacs. P. multocida was isolated from oropharynx of three of the 12 week-old and two of the 16 week-old chickens, 24 hours post-infection but not from the 4 and 8 week-old chickens. There were no gross pathological lesions or P. multocida recovered from the control birds from all the chicken age groups.

Weight changes between control and P. multocida infected chicken. The infected birds in all the age groups gained significantly $(\mathrm{P}<0.05)$ less weight than the control birds (Table 2).

Age susceptibility in family ducks ${ }_{+}$Clinical signs in ducks in relation to age group All infected ducks except three (one 12 week old and two 16 week old) expressed clinical signs of fowl cholera at some point during the 14 days observation. No mortalities were 
recorded. The frequency of all signs, except diarrhea and fever, varied significantly

between age groups (Table 1)

The eight week old ducks expressed significantly more clinical signs (188)

Formatted: Indent: First line: $36 \mathrm{pt}$

compared to the other age groups. This was also the case for the four week old ducks

(117). The susceptibility among the 12 (80) and 16 (83) weeks old ducks was equal.

Moreover, the number of severe signs was highest among the 8 week old birds (Table 2)

Control birds of all ages did not show any clinical sign throughout the observation period.

Deleted: The 8 week old ducks expressed significantly more clinical signs (188) than the other age groups followed by the 4 week old ducks (117).

Gross lesions and P. multocida re-isolation from ducks. On post mortem examination

Deleted: $I$ two 4 week-old ducks had remnants of fibrin and fibrous strands on the air sacs. One of these had pericarditis and perihepatitis. Eight of the 8 week-old ducks had remnants of fibrin and fibrous strands on the air sacs, two had fibrosis on the lungs and another two had necrotic spots on spleen and another on the liver. Two of the 12 week-old ducks had remnants of fibrin and fibrous strands on the air sacs; while four of the 16 week-old ducks had remnants of fibrin on the air sacs; one of these had necrotic spots on the liver. P. multocida was isolated from 4 of the 8 week-old and 2 of the 16 week-old ducks and one each from the 4 and 12 week-old ducks, respectively, 24 hours post infection. There were no gross pathological lesions or P. multocida recovered from the control birds from all the age groups. 
Weight changes between controls and P. multocida infected ducks The infected birds in all the age groups gained less weight than the infected birds. However, statistically the difference was only significant in the 8,12 and 16 week-groups (Table 2).

\section{Transmission of $\boldsymbol{P}$. multocida from seeder to sentinel chicken and ducks The $_{*}$} demonstration of $P$. multocida in both oropharyngeal and cloacal swabs among carrier animals is an indication that birds sharing drinking water and feed can easily transmit bacteria to each other. Therefore we decided to investigate the possibility of contact transmission between chicken and ducks (and vice versa) kept together, a conditions often seen in developing countries.

$\mid$ P. multocida was re-isolated on day one post infections from 5 out of the 6 birds of each species that were inoculated (seeder birds). This was taken as an indication that the experimental infection was successful and that they could shed the P. multocida organisms. Table 3 summarizes the daily $P$. multocida recoveries from swabs collected from sentinel birds after mixing with the seeder birds. Within 24 hours $4 / 5$ chickens and $2 / 5$ ducks had $P$. multocida organisms recovered from their swabs. By the $3^{\text {rd }}$ day, all the five sentinel chickens examined and 3/5 sentinel ducks examined had P. multocida organisms. All the five sentinel ducks were infected by the $6^{\text {th }}$ day after mixing the sentinel and seeder. The number of infected sentinel chicken declined up to day 14 . The chickens appeared to clear the $P$. multocida organisms from their oropharynx and cloacae faster than the ducks. 
$\mid$ No birds died during the experiment, but all birds, i.e. seeder and sentinel birds,

expressed clinical signs and pathological lesions of fowl cholera at one or more point during the study. The gross lesions observed were fibrino-purulent necrotic lesions on lungs and airsacs from day-three after mixing. Thereafter, the lungs and airsacs had fibrotic spots and remnants of insissipated fibrin on their abdominal airsacs appearing up to day 7. Duck had more severe lesion than chicken. No bacterial isolates were recovered from internal organs of these birds.

Discussion

This study has confirmed that family chickens and duck kept under scavenging conditions, which is very common production system in developing countries, may be healthy carriers of $P$. multocida. The isolates obtained were characterized as $P$. multocida subsp. multocida, P. multocida subsp. gallicida and $P$. multocida subsp. septica. Contrary to this, Muhairwa et al. (2001) reported only P. multocida subsp. multocida from healthy chickens. The organisms were recovered from both the oropharyngeal and cloacal swabs, but more frequently from the oropharyngeal, as reported by others (Lee et al., 2000). Systematic investigation on the occurrence of $P$. multocida has previously been carried out on healthy village chickens (Curtis and Ollerhead, 1981; Muhairwa et al., 2001) but not on traded (slaughter and live market) family chickens and ducks. In this study, the isolation rate was higher from both family chickens and ducks than previously reported (Muhairwa et al. 2001), especially, the isolation rate was high among birds 
sampled at markets and slaughterhouses. This is an indication that transport and handling in relation to marketing or slaughter may cause a $P$. multocida to increase in numbers in low level carrier birds. When evaluating this results, however, one should bare in mind that only seven ducks were tested a market/slaughter.

The degree of $P$. multocida susceptibility is variable among different types of birds and different age groups within a type (Heddleston and Wato 1965; Rhoades and Rimler, 1989). Challenge of chicken using 16 and 45 week-old New Hampshire chickens and 2 different $P$. multocida serotypes showed that mature chickens were more susceptible than the young chickens (Heddleston 1962). Similarly, an unintentional challenge of large numbers of birds with a highly virulent strain of $P$. multocida via a contaminated vaccine demonstrated no mortalities in chicken of 9-16 weeks of age while older birds died due to fowl cholera (Hungerford 1968). Taken together this has lead to the general acceptance that chicks and young growers are less susceptible to fowl cholera than older birds. The birds used in the present study are of not well defined types and cannot be referred to any of the known breeds. Msoffe et al. (2005) have characterized indigenous chickens from Tanzania by microsatellite DNA typing. They were divided into distinct genotypes, which correlated with ecotype. We assume the types used in the current study are of a similar diverse population structure, and hence genetic differences may have existed between the different groups. No genotyping was performed on birds used in the present study. Birds were selected to represent the diversity in the study area and although grouping was done in a randomized way, this did not ensure an equal distribution of ecotypes in all experiments. This must be taken into account when evaluating the results of the study. 
The attack criterion used in the studies mentioned above was mortality, as the $P$. multocida strains were lethal to the chicken. No studies have been done using a less virulent strain, expressing clinical signs but no mortality, and no observation have been done on the undefined breeds of birds kept in less intensive production systems in developing countries. In the present study, age susceptibility in chicken with respect to $P$. multocida infection was based on the number and severity of clinical signs. Twelve week-old family chickens had more clinical signs that were more severe than those of the 8,16 and 4 week-old chickens. The study confirms that different age groups have variable susceptibility to $P$. multocida as previously reported (Heddleston, 1962; Heddleston and Watko, 1965; Rhoades and Rimler, 1989), however, unlike those studies, age susceptible peaked in birds at 12 week. This does not rule out that even older birds, such as 35 week old ones, may be more susceptible than the age groups tested in the present study, however, it seems unlikely since susceptibility declined from 12 to 16 weeks. Further studies are needed to elucidate this. Clinically it was possible to pick manifestation of fowl cholera in family chicken mainly between day one to day five post infection with signs that were similar to those reported by others for intermediate to chronic fowl cholera (Gooderham, 1999; Christensen and Bisgaard, 2000; Glisson et al., 2003).

Deleted: $\mathbb{I}$

The difference between this study and previous reports could be due to both the strain of $P$. multocida used and the breed of chickens. Mortality is not a typical outcome of fowl cholera in family chicken, and we purposely looked for a strain that produced clinical and pathological signs of chronic fowl cholera. The type strain P. multocida NCTC $10322^{\mathrm{T}}$ was originally isolated from a pig, but, as documented in the present 
study, it produces clinical signs in chicken. It remains to be shown whether a more virulent strain may have different age susceptibility in the family chickens and similarly whether a less virulent strain results in different age susceptibility in commercial chicken than a highly virulent strain.

The findings of age susceptibility in ducks agree with the results of previous studies (Hunter and Wobeser, 1980), who demonstrated that birds younger than 11 weeks were more prone to infection. We demonstrated that 8 week-old village ducks had more severe clinical signs, followed by 4 week-old ducks in susceptibility to $P$. multocida. This means that age susceptibility most likely peaks somewhere between 4 and 11 weeks based on all available information. As in chicken this may depend on both breed and strain used for challenge, and further studies are needed to give the full picture. Clinically fowl cholera could be picked between day one to five in ducks with signs similar to those mentioned for chicken above.

One of the effects of fowl cholera is decrease in the feed efficiency as previously reported among affected turkeys (Morris et al., 1989) and ducks (Faddoul et al, 1967). Also older chickens sacrificed at 19 days after exposure to $P$. multocida were found to be emaciated (Heddleston and Watko, 1965). In the current study, all infected chickens and duck groups, except the 4 week old ducks, had significant weight losses compared to the control groups of their corresponding age. The weight loss was more pronounce in the elder than the younger groups, despite less clinical symptoms, possibly reflecting a higher growth rate at these ages.

Chicken and ducks infected with the low virulent strain of $P$. multocida passed the infection on to non-infected sentinel birds. The chickens appeared to clear the $P$. 
multocida organisms from their oropharynx and cloacae faster than the ducks, but the reason for this is not obvious. The intra-tracheal inoculation of chickens and ducks with P. multocida, although being artificial compared to natural infections, ensured that seeder birds were infected with the bacteria. Having the ducks and chickens sharing the same feed and water simulated the field set ups under scavenging conditions, and ensured contact between the birds. On mixing, chickens were found to peck on the bills of ducks and other chickens in an attempt to remove feed stuck around mouth and head areas. This behavior may tend to facilitate fast transmission of bacteria and other infections located in the upper respiratory tract, especially those that could be emitted through eye, nasal and mouth discharges, like P. multocida. The clinical signs and gross lesions observed in this study are similar to those reported by others (Christensen and Bisgaard, 2000;

Glisson, et al., 2003). This, together with the frequency of isolation of P. multocida from ducks and chickens in contact with the infected chickens or ducks, confirms that this bacterium was successfully transmitted from chickens to ducks. Similar scenario may be happening in market places and at farm level during scavenging, and disease control strategies should target both species of birds in field settings to be effective. The crosstransmission may play a role in the maintenance and propagation of $P$. multocida organisms at village level.

Despite the diversity and magnitude of clinical signs observed in both chicken and Deleted: II ducks in the different studies, very few pathological lesions were observed at post mortem, after the study period, except remnants of fibrin, and fibrous strands in form of fibrotic scars on the lungs and airsacs indicating chronic process. This indicates that the birds controls the low virulent strain of $P$. multocida early in the infection process. 
Bacterial isolates were recovered only in a few animals from their oropharynx shortly after infection, however, one must bare in mind, that especially in the age susceptibility study, bacterial isolation was only attempted to prove that challenge had worked and then from internal organs after 14 days. Isolation of P. multocida is difficult. The rate of isolation can be improved by passing of extracts of clinical samples through mice (Muhaiewa et al., 2001). This also proved the case in our study of carrier animals.

However, mice challenge was not attempted in the study of age susceptibility and cross transmission, and we may have overlooked bacteria in low concentration in the internal organs.

In conclusion, this study has documented the occurrence of $P$. multocida among Deleted: II Formatted: Indent: First line: $36 \mathrm{pt}$ healthy-appearing family poultry in tropical setting, and demonstrated that age susceptibility is highest in 12 week old family chicken and 8 week old family ducks when challenges with a low virulent strain of $P$. multocida. It has further demonstrated that cross transmission of fowl cholera may happen between family ducks and chickens and vice versa.

Acknowledgements

Deleted: ACKNOWLEDGEMENTS

The Chairman of Department of Veterinary Pathology, Microbiology and Parasitology is thanked for allowing us to use the laboratory facilities and the staff, especially Zackary M. Wamuri, Justus N. Matata, Henry M. Kinyua, Mary Wanjiru, John Mungai, Patrick Wahome and Samuel Indeche are thanked for technical help. The late Professor Mugera 
is thanked for constructive discussion of the work described in this paper. The study was financed by Danish International Development Agency (DANIDA) through the ENRECA program "Improving the Health and productivity of the Rural Poultry in Africa (IHEPRUCA)"'(DANIDA grant 104.Dan.8.f and University of Nairobi grant no. 500-661092). 
Refferences

Ampnsin, A., Wellehan, J. F. X., Li, L. L-., Laber, J. \& Kapur, V. (2002). DNA finger printing of Pasteurella multocida recovered from avian sources. Journal of Clinical Bermudez, A.J. \& Stewart-Brown, B. (2003). Disease prevention and Diagnosis. In, Y.M. Saif, H.J. Barnes, J.R. Glisson, A.M. Fadly, L.R. McDougald \& D.E. Swayne. (2003).

Diseases of poultry, $11^{\text {th }}$ edn. (pp 17-54). Ames: Iowa state press
Bisqaard, M. \& Mutters, R. (1986). Characterization of some previously unclassified Pasteurella spp. obtained from the oral cavity of dogs and cats and description of a new species tentatively classified with the family Pasteurellaceae Pohl 1981 and provisionally called Taxon 16. Acta Pathologica, Microbiologica et Immunologica Scandinavica, 94, $177-184$.

Chr|stensen, J. P. \& Bisgaard, M. (2000). Fowl cholera. Revue Scientifique et Technique Office International des Epizooties, 19, 626-637.

Curtis, P.E. \& Ollerhead, G.E. (1981). Investigation to determine whether healthy chicken and turkeys are oral carriers of Pasteurella multocida. Veterinary Record, 108, 206-207.

Faddoul, P.G., Fellows, G.W. \& Baird, J. (1967). Pasteurellosis in wild birds in Massachusetts.

Avian Diseases, 11, 413-418.

Glisson, J.R., Hofacre, C.L. \& Christensen, J.P. (2003). Fowl cholera. In, Y.M. Saif, H.J. Barnes, J.R. Glisson, A.M. Fadly, L.R. McDougald and D.E. Swayne (2003), Diseases of | Poultry, $11^{\text {th }}$ edn. (pp. 658-675). Ames: Iowa state press
Formatted: Indent: Before: $-27 \mathrm{pt}$, Hanging: $55.35 \mathrm{pt}$

Deleted: q
Formatted: Indent: Hanging: 28.35 $\mathrm{pt}$
Deleted: $\mathbb{\text { II }}$

Formatted: Indent: Before: -28.35 pt, Hanging: $28.35 \mathrm{pt}$

Deleted:

Formatted: Indent: Before: $0 \mathrm{pt}$

Deleted: $\mathbb{T}$

Formatted: Indent: First line: 9 pt Deleted:

Formatted: Indent: Before: $0 \mathrm{pt}$ Deleted:

Deleted: II

Formatted: Left, Indent: Before: $-28.35 \mathrm{pt}$, Hanging: $28.35 \mathrm{pt}$, Line spacing: Double

Deleted: $\mathbb{\text { II }}$

Deleted: .II 
Godderham, K.R. (1999). Avian Pasteurellosis and Pasteurella like organisms. In F. Jordan, M. Pattison, D. Alexander, \& T. Faragher (1999), Poultry Diseases, $5^{\text {th }}$ edn (pp 131-137).

Heddleston, K.L. (1962). Studies

Heddleston, K.L. (1962). Studies on pasteurellosis. V. Two immunogenic types of Pasteurella multocida associated with fowl cholera. Avian Diseases, 6, 315-321,

Heddleston, K.L. \& Watko, L-P. (1965). Fowl cholera: Comparison of serologic and immunologic responses of chickens and turkeys. Avian diseases, 9, 367-376.

Hungerford, T.G. (1968). A clinical note on avian cholera. The effect of age on the

susceptibility of fowls. Australian Veterinary Journal, 44, 31-32.

Hunter, B. \& Wobeser, W. (1980). Pathology of experimental avian cholera in Mallard ducks.

Avian Diseases, 24, 403-414

Lee C.W., Wilkie, I.W., Townsend, K.M. \& Frost, A.J. (2000). The demonstration of $P .^{4}$ multocida in the alimentary tract of chickens after experimental oral infection. Veterinary

Microbiology, 72, 47-55,

Mouris, M.P., Thayer, S.G. \& Fletcher, O.J. (1989). Characteristics of fowl cholera out breaks in turkeys in Georgia in 1986. Avian Diseases, 33, 213-218.

Msqffe, P,L.M., Mtambo, M.M.A., Minga, U.M., Juul-Madsen, H.R. \& Gwakisa, P.S. (2005). Genetic structure among the local chicken ecotypes of Tanzania based on microsatellite DNA typing. African Journal of Biotechnology, 4, 768-771.

Muhairwa, A. P., Mtambo, M.M.A., Christensen, J.P. \& Bisgaard, M. (2001). Occurrence of Pasteurella multocida and related species in village free ranging chickens and their animal contacts in Tanzania. Veterinary Microbiology, 78, 139-153,

Mutters, R., Ihm, P., Pohl, S., Frederiksen W. \& Mannheim, W. (1985). Reclassification of the genus Pasteurella Trevisan 1887 on the basis of deoxyribonucleic acid homology, with
Formatted: Indent: Hanging: 28.35 $\mathrm{pt}$

Deleted: II

Formatted: Normal, Indent: Hanging: 28.35 pt, Don't adjust right indent when grid is defined, Don't allow hanging punctuation, Don't adjust space between Latin and Asian text, Don't adjust space between Asian text and numbers

Deleted: $\mathbb{1}$

Formatted: Body Text Indent, Indent: Hanging: 28.35 pt, Automatically adjust right indent when grid is defined, Allow hanging punctuation, Adjust space between Latin and Asian text, Adjust space between Asian text and numbers

Deleted: $\mathscr{I}$

Formatted: Indent: Hanging: 28.35 pt

Deleted: II

Formatted: Indent: Hanging: 28.35 pt, Don't adjust space between Latin and Asian text, Don't adjust space between Asian text and numbers

Deleted: $\mathscr{I}$

Formatted: Justified, Indent: Hanging: 28.35 pt, Don't adjust right indent when grid is defined, Line spacing: 1.5 lines, Don't allow hanging punctuation, Don't adjust space between Latin and Asian text, Don't adjust space between Asian text and numbers

Deleted: II

Formatted: Indent: Hanging: 28.35 pt

Deleted: II

Deleted: II

Formatted: Indent: Hanging: 28.35 pt, Automatically adjust right indent when grid is defined, Allow hanging punctuation, Adjust space between Latin and Asian text, Adjust space between Asian text and numbers

Deleted: II

Formatted: Indent: Hanging: 28.35 pt 
proposal for the new species Pasteurella dagmatis, Pasteurella canis, Pasteurella stomatis, Pasteuella anatis, and Pasteurella langaa. International Journal of Systematic

Bacteriology, 35, 309-322.

Petersen, K.D., Christensen, J.P., Permin, A. \& Bisgaard, M. (2001). Avirulence of $P$. multocida subsp. multocida isolated from outbreaks of fowl cholera in wild birds for

domestic poultry and game birds. Avian Pathology, 30, 27-31.

Deleted: II

Peh ivanoglu, F., Morishita, T.Y., Aye, P.P, Porter Jr., R.E.. Angrick, E.J., Harr, B.S.

Deleted: $\mathscr{T}$

Formatted: Indent: Hanging: 28.35 pt, Automatically adjust right indent when grid is defined, Allow hanging punctuation

Formatted: Indent: Hanging: 28.35 pt

\&.Nersessian, B. (1999). The effect of route of inoculation on the virulence of raptorial

Pasteurella multocida isolates in Pekin ducks (Anas platyrhyrchos). Avian Diseases, 43,

$116-121$

Deleted: $\mathbb{1}$

Rholades, K.R. \& Rimler,R.B. (1989). Fowl cholera. p. 95-113. In, C. Adlam \& J.M. Rutter

(1989), Pasteurella and pasteurellosis (pp. 95-113), London: Academic press.

Deleted: II

Salami, J.O., Egbulem, B.N., Kwaga, J.K.P., Yusufu, H.I. \& Abdu, P.A. (1989). Diseases

diagnosed in poultry in Kaduna, Nigeria (1981 to 1985). Bulletin of Animal Health and

Production in Africa, 37, 109-114. 
Table 1: Comparison of clinical signs in chickens and ducks of different age groups

Formatted: Font: Bold infected with $\mathrm{P}$. multocida.

Formatted: Font: Italic

\begin{tabular}{|c|c|c|c|c|c|c|c|c|}
\hline \multirow{3}{*}{$\begin{array}{l}\text { Clinical } \\
\text { Signs }\end{array}$} & \multicolumn{8}{|c|}{ Number of Clinical Observations per age group } \\
\hline & \multicolumn{4}{|c|}{ Age group of chicken (weeks) } & \multicolumn{4}{|c|}{ Age group of ducks (weeks) } \\
\hline & $\overline{4}$ & 8 & 12 & 16 & 4 & 8 & 12 & 16 \\
\hline Depression & 55 & 63 & 79 & 42 & 15 & 13 & 6 & 4 \\
\hline $\begin{array}{l}\text { Nervous } \\
\text { tics }\end{array}$ & 0 & 3 & 13 & 21 & 0 & 0 & 0 & 0 \\
\hline $\begin{array}{l}\text { Ruffled } \\
\text { feathers }\end{array}$ & 36 & 61 & 74 & 27 & 15 & 13 & 6 & 4 \\
\hline Sneezing & 13 & 27 & 36 & 20 & 3 & 25 & 16 & 14 \\
\hline Ataxia & 1 & 6 & 14 & 4 & 0 & 8 & 0 & 0 \\
\hline $\begin{array}{l}\text { Nasal } \\
\text { discharges }\end{array}$ & 3 & 6 & 34 & 1 & 16 & 58 & 32 & 42 \\
\hline Dyspnoea & 4 & 17 & 16 & 13 & 22 & 26 & 1 & 3 \\
\hline $\begin{array}{l}\text { Mouth } \\
\text { discharges }\end{array}$ & 0 & 3 & 6 & 2 & 3 & 10 & 2 & 1 \\
\hline Diarrhoea & 10 & 5 & 14 & 9 & 2 & 1 & 0 & 0 \\
\hline Cyanosis & 0 & 0 & 3 & 1 & & & & \\
\hline Rales & 22 & 24 & 8 & 2 & 15 & 2 & 0 & 0 \\
\hline Fever & 29 & 53 & 60 & 38 & 26 & 23 & 15 & 14 \\
\hline $\begin{array}{l}\text { Head } \\
\text { scratching }\end{array}$ & 0 & 4 & 15 & 7 & 0 & 0 & 1 & 0 \\
\hline Coughing & 0 & 0 & 0 & 0 & 0 & 7 & 1 & 1 \\
\hline $\begin{array}{l}\text { Eye } \\
\text { discharges }\end{array}$ & 0 & 0 & 0 & 0 & 0 & 2 & 0 & 0 \\
\hline
\end{tabular}

\# The frequency of clinical signs varied significantly $(\mathrm{p}<0.05)$ between age groups of chicken, except for mouth discharges, diarrhea and cyanosis. The frequency of clinical signs varied significantly $(\mathrm{p}<0.05)$ between age groups of ducks except for diarrhea and fever. 
Table 2: Number of clinical signs observed during 14 days in different age groups of

Formatted: Font: Bold chicken and ducks infected with $\mathrm{P}$. multocida.

Formatted: Font: Italic

\begin{tabular}{|c|c|c|c|c|c|c|c|}
\hline \multirow[t]{2}{*}{ Age group } & \multicolumn{4}{|c|}{$\begin{array}{c}\text { Number of signs at days post } \\
\text { infection }\end{array}$} & \multirow{2}{*}{$\begin{array}{l}\text { Total } \\
\text { number } \\
\text { of } \\
\text { Signs }^{\dagger}\end{array}$} & \multirow[t]{2}{*}{$\begin{array}{l}\text { Severity } \\
\text { of signs }{ }^{\#}\end{array}$} & \multirow{2}{*}{$\begin{array}{l}\text { Average weight } \\
\text { loss compared to } \\
\text { control group of } \\
\text { same age }(\mathrm{g})\end{array}$} \\
\hline & $1-3$ & $4-6$ & $7-9$ & $10-14$ & & & \\
\hline Chicks - 4 & 41 & 42 & 44 & 46 & $173^{\mathrm{a}}$ & $82 / 63 / 28$ & $14.1^{*}$ \\
\hline Ducks - 4 & 51 & 39 & 12 & 16 & $117^{\mathrm{e}}$ & $72 / 22 / 23$ & 7.5 \\
\hline Chicks -8 & 51 & 69 & 63 & 89 & $272^{\mathrm{b}}$ & $143 / 70 / 59$ & $20.0^{*}$ \\
\hline Ducks - 8 & 68 & 53 & 37 & 30 & $188^{\mathrm{f}}$ & $93 / 46 / 49$ & $12.3^{*}$ \\
\hline $\begin{array}{l}\text { Chicks - } \\
12\end{array}$ & 97 & 93 & 81 & 101 & $372^{c}$ & $217 / 90 / 65$ & $25.7^{*}$ \\
\hline Ducks- 12 & 25 & 28 & 14 & 13 & $80^{\mathrm{d}}$ & $50 / 12 / 18$ & $28.3^{*}$ \\
\hline Chicks -16 & 49 & 45 & 37 & 56 & $187^{\mathrm{a}}$ & $116 / 47 / 24$ & $166.9^{*}$ \\
\hline $\begin{array}{l}\text { Ducks - } \\
16\end{array}$ & 27 & 20 & 17 & 19 & $83^{\mathrm{d}}$ & $41 / 20 / 22$ & $28.8^{*}$ \\
\hline $\begin{array}{l}\text { Control all } \\
\text { ages }\end{array}$ & 0 & 0 & 0 & 0 & 0 & $0 / 0 / 0$ & NA \\
\hline
\end{tabular}

$\dagger$ Groups of chicken that show significantly different number of clinical signs are indicated with different letters $(a, b, c)$, while statistically significant groups of ducks are indicated by letters d,e,f.

\# Severity of signs are listed in the order: mild/moderate/severe signs (see material and methods)

$* \mathrm{p}<0.05$ compared to control groups

NA: non applicable 
Table 3: Daily isolation of $\mathrm{P}$. multocida organisms from sentinel birds mixed with infected birds

\begin{tabular}{lll}
\hline Days post & Number of $P$. multocida positive sentinel birds $(\mathrm{n}=5)$ \\
contact infection & Chicken to ducks & Ducks to chickens \\
\cline { 2 - 3 } 1 & 2 & 4 \\
2 & 3 & 4 \\
3 & 3 & 5 \\
4 & 3 & 3 \\
5 & 3 & 3 \\
6 & 5 & 4 \\
7 & 4 & 4 \\
8 & 3 & 3 \\
9 & 3 & 2 \\
10 & 3 & 2 \\
11 & 4 & 2 \\
12 & 3 & 2 \\
13 & 2 & 0 \\
14 & 3 & 1 \\
\hline N / 70 possible & 44 & 39 \\
\hline & &
\end{tabular}

Formatted: Font: Bold

Formatted: Font: Italic

Formatted: Font: Italic

Formatted: Font: Not Bold

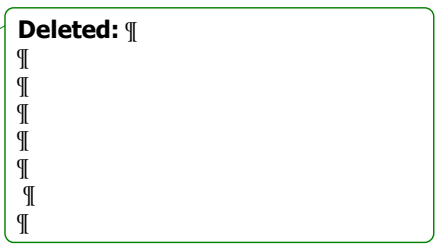

